\title{
Plummer -Vinson Syndrome: Diagnosis by Barium Esophagogram Studies May Still Be Important in the Shortage of Endoscopic Sources
}

\author{
Rabia Ergelen ${ }^{1,2}$, Mustafa Kaymakci ${ }^{1,3}$ and Hakan Akin ${ }^{1,4 *}$ \\ ${ }^{1}$ Balikesir State Hospital, Balikesir, Turkey \\ ${ }^{2}$ Marmara University Department of Radiology, Istanbul, Turkey \\ ${ }^{3}$ Department Ear Nose and Throat Private Esentepe Hospital, Istanbul, Turkey \\ ${ }^{4}$ Istinye University Liv Hospital Department of Gastroenterology, Istanbul, Turkey \\ *Corresponding author: Hakan Akin, Medicine and Gastroenterology, Istinye University Liv Hospital Department of \\ Gastroenterology, Istanbul, Turkey
}

\section{ARTICLE INFO}

Received: 絊 July 17, 2021

Published: 蔧 August 02, 2021

Citation: Rabia Ergelen, Mustafa Kaymakci, Hakan Akin. Plummer -Vinson Syndrome: Diagnosis by Barium Esophagogram Studies May Still Be Important in the Shortage of Endoscopic Sources. Biomed J Sci \& Tech Res 37(4)-2021. BJSTR. MS.ID.006039.

Keywords: Plummer-Vinson Syndrome; Iron Deficiency; Dysphagia; Esophageal Web; Barium Esophagogram; Endoscopy

\begin{abstract}
Background: Plummer-Vinson syndrome (PVS), association of iron deficiency with dysphagia due to proximal esophageal web, is still a relevant cause of dysphagia in Western societies as well as developing countries. This prospective study aims to emphasize PVS and iron deficiency prevalence in patients with dysphagia, evaluate utility of barium esophagograms in diagnosis of esophageal webs and assess efficacy of endoscopic treatment using a valid dysphagia scaling system.
\end{abstract}

Methods: Patients presenting with esophageal dysphagia were graded by Dysphagia Outcome and Severity Scale (DOSS) and 2809 adults underwent barium esophagogram studies prior to endoscopic examination. Esophageal stenosis ratios, prestenotic dilatation and diameter of web openings were measured from esophagograms. Blood samples were obtained for anemia work-up. Patients were reassessed by DOSS after endoscopic dilation therapy.

Results: Ninety-nine out of 2809 esophagograms (3.5\%) depicted esophageal webs all of which were endoscopically confirmed. PVS cases were predominantly premenopausal women. Mean esophageal stenosis ratio was $40.1 \%$. There was a weak inverse correlation between prestenotic dilatation and diameter of web opening (rho=- 0.350, p=0.001). There were no associations between hematologic parameters and fluoroscopic measurements. There was no correlation between pretreatment DOSS scores and stenosis ratios (rho $=-0.221, \mathrm{p}=0.127$ ). All patients did benefit from endoscopic dilation and iron supplementation.

Conclusion: We could not find any correlation between hematologic data and fluoroscopic measurements. All patients benefited from endoscopic dilation therapy with very low complication ratio. Although current trends favor endoscopy as the initial test for dysphagia, barium studies may still remain as an initial step in the diagnostic algorithm of dysphagia, especially in the shortage of endoscopic unit sources.

\section{Introduction}

Plummer-Vinson syndrome (PVS), also known as PatersonKelly syndrome and sideropenic dysphagia, is the association of iron deficiency with dysphagia due to proximal esophageal web.
[1] Historically, patients had been observed to be predominantly middle-aged or elderly Caucasian women of Northern European origin. [2] In recent years, due to nutritional status improvements 
and lower iron deficiency prevalence, there has been only anecdotal reports from populations in which PVS had been originally described. But PVS is still prevalent in developing countries affecting both genders, all age groups, and ethnicities. [3] Recognition of PVS is important because, apart from being a more prevalent cause of treatable dysphagia in certain populations, it represents a precancerous condition with increased risk of squamous cell carcinoma of pharynx and esophagus. PVS may also be a remote manifestation of concomitant, but undetected, pathologic processes. [4,5] This study presents a large series of PVS verified by barium esophagogram and treated with esophagoscopic intervention. Epidemiologic, clinical, and biochemical aspects of PVS are reviewed and diagnostic algorithm is reassessed based on work-up results reported from within a larger series of dysphagia cases.

\section{Materials and Methods}

This prospective study was conducted between June 2008 and September 2011 as a collaboration among radiology, otorhinolaryngology, and gastroenterology clinics of a district-level referral hospital for rural health centers serving a population of 1.1 million people. In that area, only one gastroenterology doctor was serving at that time. So, there was a shortage for reaching endoscopic unit services. Patients presenting with dysphagia were evaluated in gastroenterology and otorhinolaryngology clinics by detailed history of symptoms and thorough physical examination. Patients with symptoms suggesting esophageal dysphagia, i.e., difficulty in transporting ingested material down the esophagus as opposed to oropharyngeal dysphagia which is the inability to initiate swallowing and failure of bolus transfer from the mouth to the esophagus, were further assessed by grading of symptoms using Dysphagia Outcome and Severity Scale (DOSS). [6] This test, a simple and practical 7-point scale for rating functional severity of dysphagia, is based on level of independence, nutrition type and diet level and has proven to have favorable inter and intra-rater reliability (Table 1). [7] A total of 2809 adult patients were referred for barium esophagogram studies to provide a roadmap prior to upper endoscopy.

Studies were performed using standard and modified barium swallow and esophagography protocols with single-contrast examinations incorporating a full column of thin-barium suspension, i.e., $40-80 \% \mathrm{w} / \mathrm{v}$, to provide optimal luminal distension and enable motility assessment. [8-10] Barium studies were examined by two radiologists experienced in performing and interpreting barium esophagograms. Radiographic diagnosis of proximal esophageal web, defined as a very short segment of acute narrowing in the barium column at cervical levels, was made by consensus of the two radiologists.

Following measurements were recorded:

a. Narrowest width of the barium column at the level of the web,

b. Maximum width of the barium column at the prestenotic dilated segment, and

c. Diameter of normal-looking esophagus distal to the web.

Table 1: Summary of dysphagia outcome and severity scale (DOSS).

\begin{tabular}{|c|c|c|c|}
\hline Level & Explanation & Diet & Intervention \\
\hline 7 & Normal & Normal & None needed. \\
\hline 6 & Within functional limits & Normal & Need extra time for meal. \\
\hline 5 & Mild dysphagia & One diet consistency restricted. & Distant supervision. \\
\hline 4 & Mild-moderate dysphagia & One or two consistencies restricted. & Intermittent supervision / cueing. \\
\hline 3 & Moderate dysphagia & $\begin{array}{c}\text { Two of more consistencies } \\
\text { restricted. }\end{array}$ & Total supervision necessary. \\
\hline 2 & Moderate-severe dysphagia & $\begin{array}{l}\text { Tolerates at least one consistency } \\
\text { safely with total assistance. }\end{array}$ & $\begin{array}{l}\text { Partial PO with maximum assistance or no PO } \\
\text { at all. } \\
\text { Non-oral nutrition necessary. }\end{array}$ \\
\hline 1 & Severe dysphagia & Unable to tolerate any PO safely. & Non-oral nutrition only. \\
\hline
\end{tabular}

Ratio of prestenotic dilatation to poststenotic normal esophagus, and stenosis ratio were derived from the measurements (Figure 1). Patients diagnosed with proximal esophageal webs, along with other patients referred for barium esophagograms, were then prepared for elective endoscopy and blood samples for anemia work-up were obtained prior to intervention. All endoscopic interventions were performed by a single operator with more than 5 years of endoscopy experience. Study was in compliance with institutional review board guidelines and written informed consent was obtained from all patients. under light sedoanalgesia, and without fluoroscopic guidance (Figure 2). Endoscopic treatment comprised insertion of a metallic guidewire through the web opening and slipping dilators, i.e., bougies with diameters $0.9-1.5$ $\mathrm{mm}$, over the guidewire and gradually increasing in size without exceeding 2 successive calibers per session. Rupture of the web was confirmed by endoscopy and esophageal biopsy samples were taken in all cases. Patients resumed regular diet the next day and were also given oral iron supplements to replenish depleted 
iron stores. Severity of dysphagia symptoms were reassessed at the fourth- and twelfth-weeks post-intervention with DOSS. Statistical analysis was performed using commercially available software (IBM SPSS Statistics for Windows, Version 21.0, IBM Corp., Armonk, NY). Categorical variables were analyzed with Pearson's Chi-Square and Fisher's Exact test. Mann-Whitney U test was used for comparison of group parameters. A logistic regression analysis determined effect of fluoroscopic modifiers on dysphagia severity scores and possible correlative associations among variables were sought by Pearson's coefficient tests. Results were evaluated within a 95\% confidence interval and significance level was set at $\mathrm{p}<0.05$.

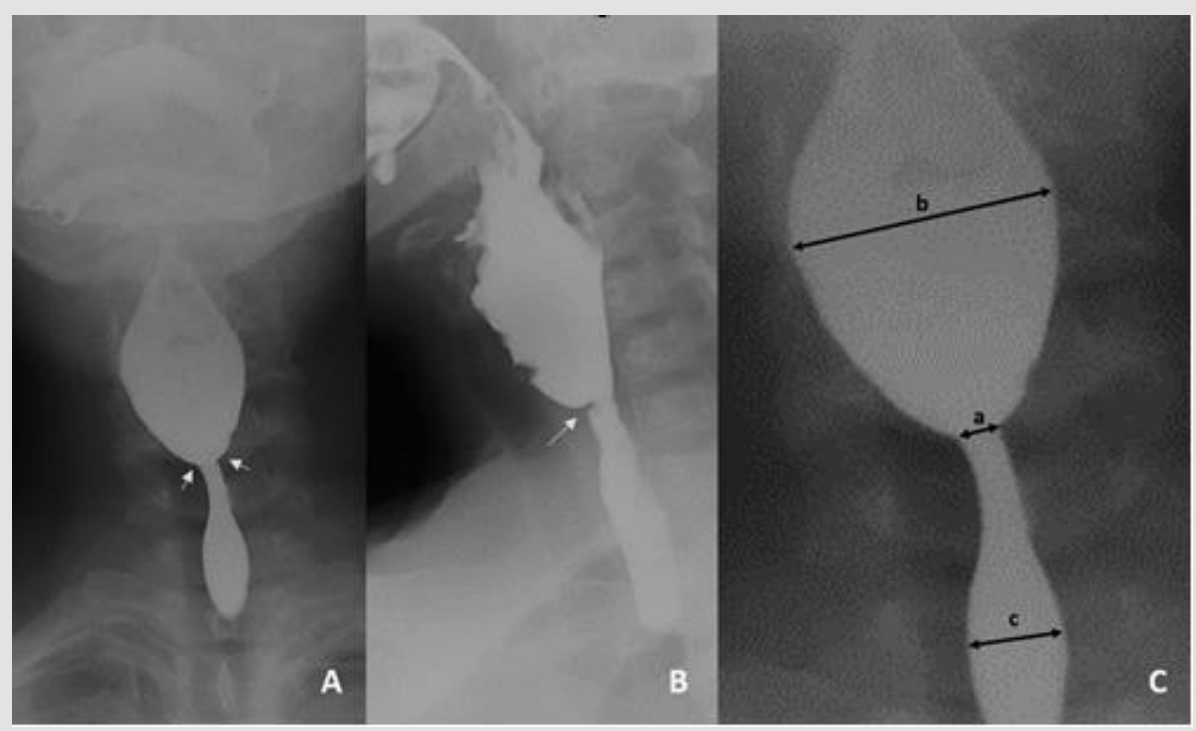

Figure 1:

a. [A, B] Anteroposterior and lateral view of barium esophagogram depicting a web (arrows) located at cervical esophagus. b. [C] Measurements on barium esophagograms (a: narrowest width of barium column, b: maximum width of the barium column at prestenotic dilated segment, and c: diameter of normal-looking esophagus distally).

Following ratios were derived from measurements: Ratio of prestenotic dilatation to poststenotic normal esophagus [=b/c], and stenosis ratio $[=(\mathrm{c}-\mathrm{a}) / \mathrm{c}]$.

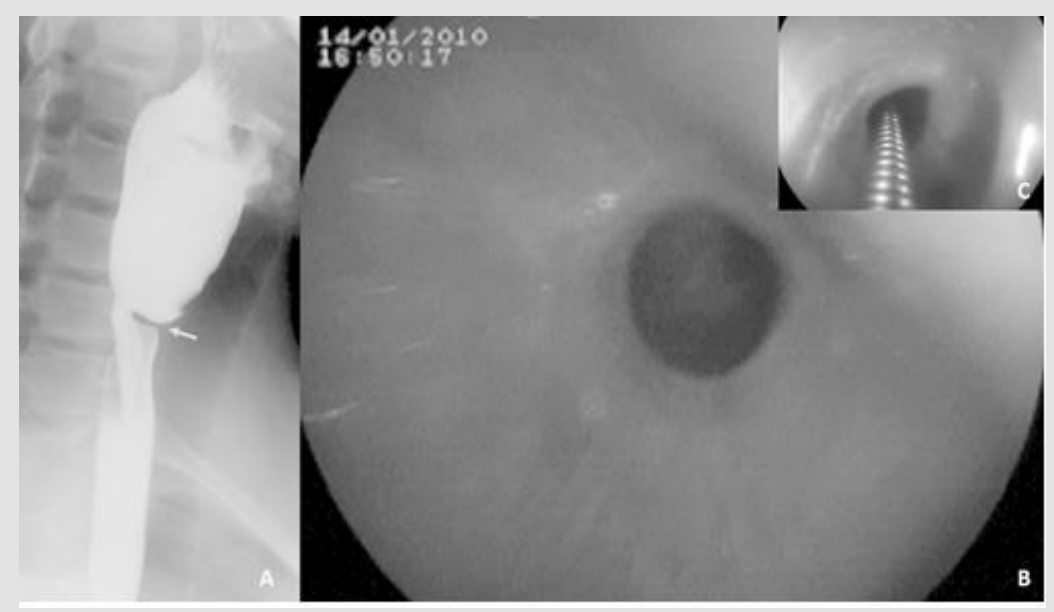

Figure 2:

A. Barium esophagogram showing a web (arrow) within cervical esophagus.

B. Endoscopy emonstrating a circumferential esophageal web just below the upper esophageal sphincter.

C. Endoscopic view during insertion of a metallic guidewire through the web opening prior to bougie dilation. 


\section{Results}

Ninety-nine out of 2809 esophagograms (i.e.,3.5\%) depicted esophageal webs all of which were later confirmed endoscopically by direct visualization as smooth, thin, grayish membranes with eccentric or central openings. Ninety-one out of 99 cases were female (92\%) and seventy-five percent of female patients were premenopausal. Patient ages ranged 18 to 84 years with a mean of $46.7 \pm 14.1$ years. Age distribution among genders were statistically insignificant. Anemia work-up revealed sideropenia, hence confirming diagnosis of PVS (Table 2). Esophageal stenosis ratios ranged from $6 \%$ to $97 \%$ (mean $40.1 \pm 23.2$ percent). Ratio of prestenotic dilatation to poststenotic normal esophagus varied between 1.04 and 3.31 (mean $1.51 \pm 0.41$ ). There was no significant correlation between the two derived ratios ( $r h o=0.198, p=0.071$ ). There was a weak, albeit statistically significant, correlation between width of prestenotic dilatation and diameter of web opening ( $\mathrm{rho}=-0.350, \mathrm{p}=0.001)$. On the other hand, there were no statistically significant associations between hematologic parameters and fluoroscopic measurements or derived ratios ( $p>0.05$ in all pairs). Pretreatment levels of dysphagia based on Dysphagia Outcome and Severity Scale (DOSS) were distributed as follows: moderate (18.4\%), mild - moderate (18.4\%), mild (26.5\%), functional (16.3\%), and normal (20.4\%). None of the patients had level 1 or 2 dysphagia.

Table 2: Anemia work-up of PVS cases.

\begin{tabular}{|c|c|c|c|c|c|c|}
\hline & Unit & Normal Values & Minimum & Maximum & Median & Mean \pm SD \\
\hline Hemoglobin & $\mathrm{g} / \mathrm{dL}$ & $\begin{array}{c}12.0-16.0^{*} \\
13.5-17.5^{* *}\end{array}$ & 7.1 & 15.9 & 11 & $11.1 \pm 2.6$ \\
\hline Hematocrit & $\%$ & $\begin{array}{c}36-47^{*} \\
38-54^{* *}\end{array}$ & 22.4 & 45.5 & 34.7 & $35.3 \pm 5.2$ \\
\hline MCV & $\mathrm{fL}$ & $78-98$ & 54.9 & 109.1 & 75.9 & $76.6 \pm 10.7$ \\
\hline Platelet count & $\mathrm{x} 1000 / \mathrm{mm}^{3}$ & $200-500$ & 27 & 587 & 274 & $285.4 \pm 109.8$ \\
\hline Serum Iron & $\mu \mathrm{g} / \mathrm{dL}$ & $\begin{array}{c}65-176^{* *} \\
50-170^{*}\end{array}$ & 10 & 142 & 47 & $51.8 \pm 35.6$ \\
\hline Serum Ferritin & $\mu \mathrm{g} / \mathrm{L}$ & $\begin{array}{l}12-150^{*} \\
12-300^{* *}\end{array}$ & 2 & 83 & 12 & $20.5 \pm 20.2$ \\
\hline TIBC & $\mu \mathrm{g} / \mathrm{dL}$ & $240-450$ & 166 & 906 & 366 & $369.8 \pm 127.5$ \\
\hline
\end{tabular}

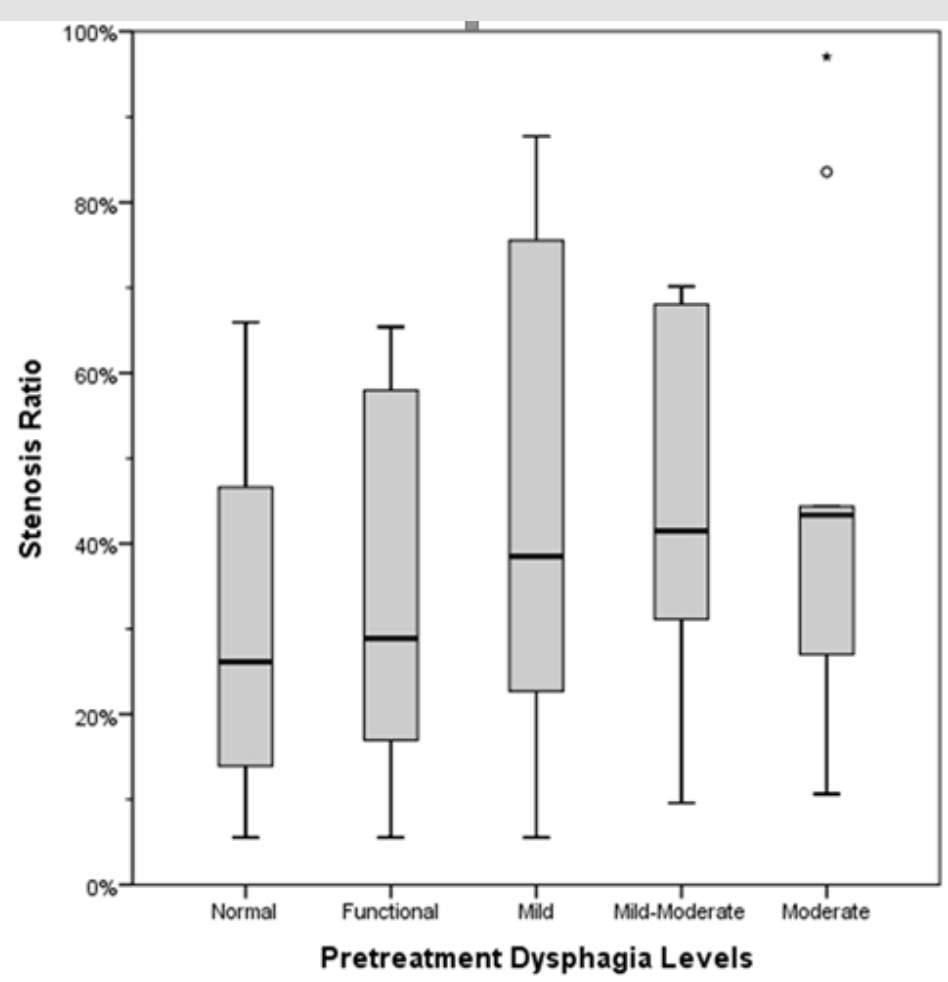

Figure 3: Boxplot chart of pretreatment dysphagia levels (based on dysphagia outcome and severity scale - DOSS) versus stenosis ratios. There was no correlation between two variables ( $r h o=-0.221, p=0.127$ ). 
Ten patients required multiple sessions of endoscopic dilation and one patient had multiple webs in her cervical esophagus. No procedural adverse events were observed. There was no significant correlation between pretreatment DOSS scores and stenosis ratios (rho=-0.221, p=0.127) (Figure 3). However, all patients, regardless of their original stenosis ratios and pretreatment DOSS scores, did benefit from endoscopic dilation and ongoing iron supplementation. Post-treatment controls documented greatly improved dysphagia symptoms with only DOSS levels 6 and 7 remaining. At the 4 th week assessment $38.8 \%$ scored level 6 and $61.2 \%$ scored level 7; whereas, at the $12^{\text {th }}$ week reassessment $14.3 \%$ remained level 6 and $85.7 \%$ scored level 7. Seventeen PVS patients had diagnoses responsible for chronic iron loss: Gastric angiodysplasia (n=1), subtotal gastrectomy $(n=9)$, coeliac disease $(n=2)$, gastric carcinoma $(n=2)$, colon carcinoma $(n=2)$ and gastric antral vascular ectasia $(n=1)$. Remaining 92\% had no underlying gastrointestinal pathology identified. One patient developed squamous cell carcinoma of the esophagus during follow-up.

\section{Discussion}

Dysphagia is an alarming symptom requiring immediate evaluation. Type of dysphagia, i.e., oropharyngeal versus esophageal, and its underlying cause, i.e., structural - anatomic as opposed to motility disorders, can be distinguished by patient's history and physical examination. Rarest subtype of esophageal dysphagia is cervical dysphagia. Apart from malignancies, this subtype of dysphagia is most commonly caused by upper esophageal

webs. $[3,11]$ Reports of PVS from populations in which it had been originally described has diminished significantly. However, PVS is still not a completely ignorable cause of dysphagia in Western societies, because certain subpopulations are at increasingly greater risk of developing chronic malnutrition. Increased elderly population along with various associated comorbidities and malignancies; increasing prevalence of extreme obesity undergoing bariatric surgery; and populations affected by inflammatory bowel diseases -still more prevalent in Europe, North America, and Australia - are prone to malabsorption states including chronic iron deficiency.[12-14] On the other hand, iron deficiency anemia due to chronic malnutrition is still prevalent in the developing world, and may present with PVS in in all age groups and both genders.

$[3,15]$ In this study, all webs detected by esophagograms have been confirmed to be associated with PVS. Our 3.5\% incidence is in agreement with the literature. However, there are studies reporting that not all upper esophageal webs cause dysphagia and even a smaller fraction is associated with PVS. An influential study by Nosher, et al. [16] reported upper esophageal webs, present in $5.5 \%$ of screening esophagograms, were not associated with dysphagia; and while overall incidence of iron deficiency anemia was increased in patients with upper esophageal webs, the difference was statistically insignificant $[3,11,16-17]$ However, the mentioned study suffers from a limitation similar to ours: Both studies cannot estimate the effect of any prior treatments to correct iron deficiency states on laboratory parameters of anemia work-up. It is highly probable that anemia states had been previously noticed in patients, and treatments such as dietary or parenteral iron supplementation, and even blood transfusions, were attempted before dysphagia became a major symptom. This, we believe, may cause underestimation of anemia - or iron deficiency states in general - as an etiologic factor for upper esophageal web formation, and might have resulted in underdiagnosis of PVS in populations prone to malabsorption states. American Gastroenterological Association has been recommending upper endoscopy as the initial diagnostic test in patients with dysphagia.

An exception to this recommendation is clinical suspicion of achalasia or oropharyngeal disorders such as upper esophageal complex strictures due to caustic ingestion, laryngectomy, or radiotherapy. In these cases, AGA recommended using barium swallow as the first diagnostic test. [18] Endoscopically, esophageal webs associated with PVS appear as smooth, thin, grayish membranes usually protruding horizontally from the anterior wall and extending laterally. [15] Webs originating from the posterior wall or encircling the lumen are less common. On the other hand, webs with pinhole sized central lumen presenting with severe dysphagia are also reported. Since these webs typically occur in proximal esophagus, they may be missed and accidentally ruptured unless the endoscope is introduced under direct visualization by an experienced endoscopist. Although such patients are relieved of their dysphagia symptoms after endoscopy, underlying chronic iron deficiency is left untreated and many associated comorbidities - such as coeliac disease, inflammatory bowel disease, pernicious anemia, thyroid diseases, Sjogren's disease and rheumatoid arthritis - may be left undiagnosed.

$[3,19]$ Underdiagnosis of PVS is not of trivial importance because it is considered not only as a precancerous lesion itself, but also as a sign of malignancy elsewhere. $[11,15,20]$ It has been proposed that barium swallow was more cost effective than upper endoscopy for diagnosis of abnormal esophageal motility, and in contrast, initial endoscopy with therapeutic intent was less costly for patients suspected with history suggesting benign obstruction. [21] On the other hand, webs associated with PVS may resolve spontaneously with iron supplementation therapy, obviating majority of endoscopic interventions. [22] Barium esophagograms are likely to provide a better roadmap prior to therapeutic interventions. In addition, the radiation dose to the patients during barium swallow studies is well within established safety limits and average patient entrance surface dose is $12.6 \pm 10 \mathrm{mGy}$ with organ equivalent dose as low as $0.20 \pm 0.0 \mathrm{mSv}$. [23] Thus, we believe, barium studies still may remain as an initial step in diagnostic algorithm of dysphagia, 
especially in the shortage of endoscopist or endoscopy unit sources which is a current reality in most parts of the developing countries even in some of the rural areas of developed ones.

Screening with barium studies prior to endoscopic intervention may not always be feasible though. There were 6 patients, all female, aged 44-55 years, who presented with acute food impaction and were treated with emergent endoscopic evacuation and, upon confirming upper esophageal webs, with endoscopic dilation in the same session. Having no pre-intervention esophagograms, these patients were excluded from the study. Anemia work-up was carried out nevertheless, and revealed iron deficiency in all patients, thus establishing the diagnosis of PVS. Dysphagia associated with PVS is typically intermittent and limited to solid foods. Patients may also complain about choking and sensation of a "lump in the throat," and less commonly, aspiration episodes. Barium esophagography is important for interdisciplinary diagnostic work-up of underlying benign esophageal pathologies in patients with globus-like symptoms who have no known disease, no history of head and neck surgery, and no thyroid pathology. [6,24-26] However, considering the relative rarity of PVS along with its predilection for middle-aged perimenopausal women and chronically ill subjects, physicians who forego esophagograms may misinterpret these complaints as symptoms of a panic disorder and such patients may suffer from public and self-stigma of mental illness.

Dysphagia Outcome and Severity Scale (DOSS) is a measure of dysphagia and functional capacity based on food intake ability and indicates the best nutritional regimen for everyone. Like other assessment tools of dysphagia, DOSS was developed to provide reliable outcome data in dysphagia research, a trend driven by managed care in recent years. [7] DOSS has good statistical concordance, but it is criticized for being regarded as somewhat subjective by some authors. This subjectivity, which is also observed in other sphagia scales, may bring interpretation bias, and hinder its utility in follow-up or decision-making. [27] In this study, all patients with esophageal webs were endoscopically treated, thus the scale was not used for clinical decision making. In addition, endoscopic treatment, due to previous experience reported in literature, was expected to result in very dramatic improvements of symptoms, with no room for subtlety or ambiguity, therefore, we believe, utility of the scale in post-treatment assessment was not compromised. This study reflects experience regarding PVS from a district-level referral hospital for rural health centers. Although serving a great hinterland of more than one million people, data obtained from such centers may suffer from lack of demographic diversity in the sample population. Since etiopathogenesis of PVS involves multiple factors including both environmental and genetic - immunologic influences, our results may be merely reflecting the local populace, thus, precluding accurate extrapolation to larger populations. Genetic underlying factors must also be further evaluated to reach universal conclusions. $[3,5,11]$
In conclusion, Plummer - Vinson syndrome must be regarded as a sign reflecting potentially serious problems in both the individual's health and the public healthcare system, and not as an ancient and extinct disease. Despite the decline in referrals for barium swallow studies due to current trends favoring endoscopy, interdisciplinary cooperation is still the key to reducing underdiagnosis of PVS. Barium studies are good in the detection of upper esophageal webs, and they provide an accurate roadmap for endoscopic intervention which substantially eases the process for both the patient and the endoscopist. Thus, barium studies are still indispensable and still may remain as an initial step in diagnostic algorithm of dysphagia, especially in the shortage of endoscopy unit sources.

\section{Conflict of Interest Statement}

All authors certify that they have no affiliations with or involvement in any organization or entity with any financial interest (such as honoraria; educational grants; participation in speakers' bureaus; membership, employment, consultancies, stock ownership, or other equity interest; and expert testimony or patentlicensing arrangements), or non-financial interest (such as personal or professional relationships, affiliations, knowledge or beliefs) in the subject matter or materials discussed in this manuscript.

\section{References}

1. Bakari G, Benelbarhdadi I, Bahije L, A El Feydi Essaid (2014) Endoscopic treatment of 135 cases of Plummer-Vinson web: a pilot experience. Gastrointest Endosc 80(4): 738-41.

2. Wynder EL, Hultberg S, Jacobsson F, Bross IJ (1957) Environmental factors in cancer of the upper alimentary tract; a Swedish study with special reference to Plummer-Vinson (Paterson-Kelly) syndrome. Cancer 10(3): 470-87.

3. Novacek G (2006) Plummer-Vinson syndrome. Orphanet J Rare Dis 1: 36.

4. Demirci F, Savas MC, Kepkep N, Vahap Okan, Mehmet Yilmaz, et al. (2005) Plummer-Vinson syndrome and dilation therapy: a report of two cases. Turk J Gastroenterol 16(4): 224-7.

5. Nagai T, Susami E, Ebihara T (1990) Plummer-Vinson syndrome complicated by gastric cancer: a case report. The Keio journal of medicine 39(2): 106-11.

6. Gasiorowska A, Fass R (2009) Current approach to dysphagia. Gastroenterol Hepatol 5(4): 269-279.

7. O’Neil KH, Purdy M, Falk J, Gallo L (1999) The Dysphagia Outcome and Severity Scale. Dysphagia 14(3): 139-145.

8. Levine MS, Rubesin SE (1990) Radiologic investigation of dysphagia. AJR Am J Roentgenol 154(6): 1157-63.

9. Levine MS, Rubesin SE, Ott DJ (1990) Update on esophageal radiology. AJR Am J Roentgenol 155(5): 933-41.

10. Eisenhuber E, Schima W, Schober E, Peter Pokieser, Alfred Stadler, et al. (2002) Videofluoroscopic Assessment of Patients with Dysphagia. American Journal of Roentgenology 178(2): 393-8.

11. Atmatzidis K, Papaziogas B, Pavlidis T, Mirelis C, Papaziogas T (2003) Plummer-Vinson syndrome. Diseases of the esophagus: official journal of the International Society for Diseases of the Esophagus / ISDE 16(2): 154-7.

12. Bonovas S, Fiorino G, Allocca M, Theodore Lytras, Argirios Tsantes, et 
al. (2016) Intravenous Versus Oral Iron for the Treatment of Anemia in Inflammatory Bowel Disease: A Systematic Review and Meta-Analysis of Randomized Controlled Trials. Medicine 95(2): e2308.

13. Inge $\mathrm{TH}$, Courcoulas AP, Jenkins TM, Marc P Michalsky, Michael A Helmrath, et al. (2016) Weight Loss and Health Status 3 Years after Bariatric Surgery in Adolescents. The New England journal of medicine 374(2): 113-23.

14. Malik TA (2015) Inflammatory Bowel Disease: Historical Perspective, Epidemiology, and Risk Factors. Surg Clin North Am 95(6): 1105-22.

15. Gultepe I, Basaranoglu M (2016) Two cases with Plummer-Vinson syndrome in the $21^{\text {st }}$ century. Turk J Gastroenterol 27(1): 81-2.

16. Nosher JL, Campbel WL, Seaman WB (1975) The clinical significance of cervical esophageal and hypopharyngeal webs. Radiology 117(1): 45-7.

17. Godino J, Wong PW (2000) A triad of troubling findings. PlummerVinson syndrome. Postgraduate medicine 108(2): 109-10.

18. Spechler SJ (1999) American gastroenterological association medical position statement on treatment of patients with dysphagia caused by benign disorders of the distal esophagus. Gastroenterology 117(1): 22933.

19. Gude D, Bansal D, Malu A (2013) Revisiting plummer vinson syndrome. Annals of medical and health sciences research 3(1): 119-21.

\section{ISSN: 2574-1241}

DOI: 10.26717/BJSTR.2021.37.006039

Hakan Akin. Biomed J Sci \& Tech Res

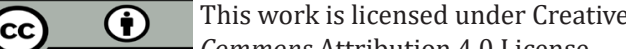

Commons Attribution 4.0 License

Submission Link: https://biomedres.us/submit-manuscript.php
20. Changela K, Haeri NS, Krishnaiah M, Reddy M (2015) Plummer-Vinson Syndrome with Proximal Esophageal Web. J Gastrointest Surg 20(5): 1074-1075.

21. Esfandyari T, Potter JW, Vaezi MF (2002) Dysphagia: a cost analysis of the diagnostic approach. The American journal of gastroenterology 97(11): 2733-7.

22. Phatak S, Redkar N, Patil MA, Kuwar A (2012) Plummer-Vinson syndrome. BMJ Case Rep 2012.

23. Sulieman A, Elzaki M, Kappas C, Theodorou K (2011) Radiation dose measurement in gastrointestinal studies. Radiat Prot Dosimetry 147(12): $118-21$.

24. Alberty J, Oelerich M (1996) [Use of high frequency cinematography in diagnosis of globus sensation]. Laryngo- rhino- otologie 75(9): 533-7.

25. Caylakli F, Yavuz H, Erkan AN, Ozer C, Ozluoglu LN (2006) Evaluation of patients with globus pharyngeus with barium swallow pharyngoesophagography. The Laryngoscope 116(1): 37-9.

26. Adachi J, Ohmae Y, Karaho T, Tetsuya Tanabe, Daisuke Mizokami, et al. (2010) Relationship between globus sensation and esophageal clearance. Acta oto-laryngologica 130(1): 138-44.

27. Sallum RA, Duarte AF, Cecconello I (2012) Analytic review of dysphagia scales. Arquivos brasileiros de cirurgia digestiva: $\mathrm{ABCD}=$ Brazilian archives of digestive surgery 25(4): 279-282.

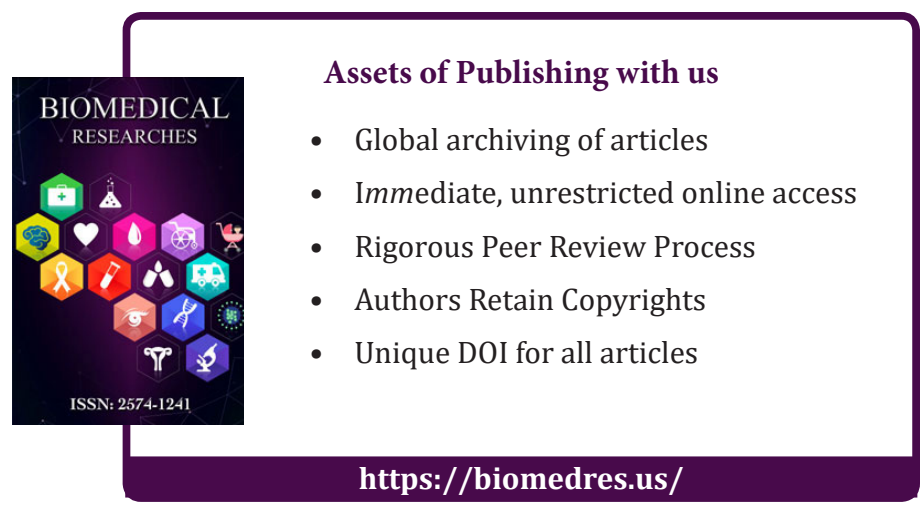

